\title{
Epidemiological study of congenital and hereditary anomalies in Sialkot District of Pakistan revealed a high incidence of limb and neurological disorders
}

\author{
Nadeem Ahmad Bhatti', Sara Mumtaz ${ }^{1,2}$, Sajid Malik ${ }^{1, *}$
}

\begin{abstract}
Background: Pakistan faces high incidence of congenital anomalies (CA) and hereditary anomalies due to various factors, including a high rate of consanguinity, early marriages, and predominance of extended families. There is a paucity of epidemiological studies that could provide a baseline for management strategies for these anomalies.

Objectives: We aimed to elucidate the pattern, as well as the clinical and genetic aspects, of CA prevalence among the general population in Sialkot District of Pakistan.

Methods: In a cross-sectional sampling design, subjects and families with a certain type of CA were recruited from hospitals and medical centers in Sialkot District. Subjects were also selected from various towns and remote villages by visiting public places. Phenotypic and descriptive data were obtained, pedigrees were constructed, and parental and demographic attributes were recorded.

Results: A total of 241 independent subjects and/or families with CA were recruited. The malformations were classified into five major and 56 minor categories. Limb defects had the highest representation $(\mathrm{n}=113$; proportion $=0.469$; $95 \%$ confidence interval $(\mathrm{CI})=0.406-0.532)$, followed by neurological anomalies $(\mathrm{n}=76$; proportion $=0.315 ; 95 \%$ $\mathrm{CI}=0.257-0.374)$. Among the limb defects, polydactyly and talipes were most prevalent while, among neurological disorders, intellectual disability and cerebral palsy were more frequent. In this cohort, sporadic occurrence was customary compared to the familial presentation $(\mathrm{n}=144$ vs 97$)$. Analyses of various attributes, such as gender differences, parental consanguinity, and paternal ages, as well as pedigree analyses, revealed marked heterogeneity among the major and minor categories of CA.

Conclusion: The pattern of anomalies witnessed in this cohort and a high occurrence of sporadic cases point to a substantial role of nongenetic etiological factors, which could be minimized by strengthening the health-care system.
\end{abstract}

Keywords: consanguinity; genetic diseases; prevalence

Congenital anomalies (CA) are a heterogeneous group of disorders that are present at or before birth. CA could be due to genetic factors, such as single gene defects and chromosomal abnormalities, or could be caused by environmental influences, which include micronutrient deficiencies, teratogen exposure, and infections [1]. A large number of CA with genetic etiology

*Correspondence to: Sajid Malik, Department of Animal Sciences, Faculty of Biological Sciences, Quaid-i-Azam University, Islamabad 45320,

Pakistan, e-mail: malik@qau.edu.pk

'Department of Animal Sciences, Faculty of Biological Sciences, Quaid-i-Azam University, Islamabad 45320, Pakistan

2Department of Biological Sciences, National University of Medical Sciences, Rawalpindi 46000, Pakistan

O Open Access. ๑ 2019 Nadeem Ahmad Bhatti et al., published by Sciendo. (c) Br-Nc-ND This work is licensed under the Creative

Commons Attribution NonCommercial-NoDerivatives 4.0 License. 
may be hereditary in nature. Every year, 303,000 children die within 4 weeks of birth due to CA, and others with long-term disabilities negatively affect families, health-care systems, and societies [2].

In developed countries, there is systematic registration of CA [1, 3, 4]. In many developing countries, however, birth defect registries are not maintained. Monitoring the malformations and establishing their sociodemographic correlates is not an easy task and is hampered by a number of factors, including inadequate infrastructure for surveillance, ascertainment biases - particularly in rare anomalies, and invasive methods of diagnosis in certain cases. In the absence of detailed epidemiological data on $\mathrm{CA}$, it is difficult to evaluate possible risk factors and to implement effective prevention and care services $[5,6]$.

The health-care system of Pakistan is facing various challenges in service delivery to the population. The majority of the population resides in rural areas, where the infrastructure of health care is present, but it is poorly maintained, in addition to lacking modern equipment and trained professionals [7]. Despite significant improvements over the past two decades in Pakistan, the infant and neonatal mortality rates remain high. In 2015, Pakistan was ranked 149th among 179 countries on the Maternal Mortality Ratio Index, which is very alarming [8]. The allocation of health-care resources, such as finance and transport, is not a need-based process in Pakistan. Furthermore, owing to the high rate of population growth, the number of health-care professionals is inadequate and the existing medical staff is untrained, underpaid, and deprived of the latest facilities for medical practice. Nonetheless, the health-care system mainly relies on private organizations, which provide more advanced facilities at high prices, and the masses cannot afford these facilities. There are massive inequalities in the accessibility of health-care services to the low-socioeconomic-status population [7-9].

According to a recent estimate, CA caused $2.34 \%$ of total deaths in Pakistan [3]. Here, the high incidence of CA has been attributed to various factors, such as maternal malnutrition, inadequate prenatal care, poor socioeconomic setup, rural origin, and a high rate of consanguinity [10-12]. In a study carried out in the Combined Military Hospital, Kharian, Hussain et al. [13] showed that CA affected 7\% of the 3,210 cases. Previously, Perveen and Tyyab [14] showed that neural tube defects were the most prevalent type of anomalies in a tertiary care hospital in Karachi and the commonest associated risk factor was consanguinity. A detailed survey of the medical literature revealed that the majority of the reports on CA were hospital based [10-13]. Such studies may have an underrepresentation of subjects from rural areas, where most of the deliveries take place at home and with the help of traditional birth attendants [15]. Due to lack of proper surveillance and documentation, the countrywide picture on the epidemiology of CA is fragmented.

The present clinico-epidemiological study on CA was carried out in the Sialkot District of Pakistan, where the majority of the population resides in rural areas.

\section{Methods}

\section{Study population}

A descriptive clinical and genetic epidemiological study on CA was carried out in the Sialkot District, which is located in the northeast of Pakistan. The district has four administrative units called "tehsils". The rural population comprises $74 \%$ of the total population, and the literacy rate is $59 \%$. Major ethnicities are the Jatts and Gujjars, while there is also a representation of Rajput, Arain, and Kashmiris. Major languages are Punjabi and Kashmiri [16]. According to the Pakistan Census of 2017, it had a population of 3.9 million. It is an industrial city and is famous for its leather, sports, and surgical products [17].

\section{Ethical considerations and sampling strategy}

The study was approved by the Ethical Review Committee of Quaid-i-Azam University, Islamabad (DFBS/2014-3278). All the data were acquired and documented in writing in the presence of family head/guardian after participants had provided informed written or formally documented verbal consent when illiterate. When a participant was below the legal age of providing consent or was incapable of providing it because of disability (deaf-mute, blind, neuromuscular defect), or otherwise incapable, a parent/guardian or literate elder provided written informed consent. They were assured before the start of the study that there would be no breaches of an ethical nature or confidentiality.

In a cross-sectional sampling design, subjects and families with CA and/or hereditary anomalies were recruited from June 2013 to December 2014. The subjects/families were recruited from District Headquarters Hospitals and Basic Health Units in different rural and urban areas of Sialkot District. Since there is no systematic record keeping in the public sector hospitals, all the cases were retrospectively recruited by the data collection team. The subjects were also recruited from public places such as community centers and rural 
gathering areas and were brought to the nearest medical center for examination. Each subject was physically examined by a local physician. The previous health record of the subjects when available was assessed by two expert health workers. The fieldwork and data collection were performed by one of the coauthors, who had had formal training in medical genetics, along with a local resource person who guided in subject recruitment. The resource persons were male nurses or paramedical staff who were familiar with the local population. The data were not collected from any rehabilitation or special education center.

\section{Classification of anomalies}

For the primary diagnosis, we relied on the assessment provided by the specialist resident doctor, and only those individuals with confirmed congenital and/or hereditary anomalies were recruited. The cases were identified with respect to the index male or female subject and were categorized as follows: (i) familial or sporadic, and (ii) isolated or syndromic. Syndromic cases were identified with respect to the more severe symptoms in the following order: neurological disorders, neuromuscular defects, musculoskeletal defects, eye/visual impairments, sensorineural/ear anomalies, and limb defects. Secondary symptoms, when present, were separately scored as associated malformations. A pedigree comprising three or more generations was drawn for each case; however, only the index subject was considered in the data analyses. Malformations of infectious or traumatic nature and subjects with poliomyelitis were not included. All the information was recorded on a structured questionnaire that was divided into three sections: the first section included demographic data; the second section dealt with various risk factors such as parental medical history (including paternal ages) and consanguinity; and the third section documented the phenotypic details of the anomalies.

The definition of CA was based on a standard coding system of the International Classification of Diseases, Tenth Revision (ICD-10), according to the primary diagnosis [18]. The corresponding definitions of each entity were searched in the Online Mendelian Inheritance in Man (OMIM) and Orphanet databases $[19,20]$. Limb defects, such as syndactyly and polydactyly, were further characterized into well-described entities [21-23]. For each CA, the proportions and 95\% confidence intervals ( $95 \%$ CIs) were estimated from the total number of anomalies. Statistical analyses were performed using MS-Excel and GraphPad Prism.

\section{Results}

\section{Sample characteristics}

A total of 241 independent subjects/families with certain types of CA were identified in this study. There were 181 $(75 \%)$ index males and $60(25 \%)$ index females (Table 1). The CA were classified into five major and 56 minor categories (Table 2). Among the major categories, limb defects had the highest representation $(n=113$; proportion $=0.337$; $\mathrm{CI}=0.278-0.397)$, followed by neurological disorders $(\mathrm{n}=76)$, musculoskeletal defects $(\mathrm{n}=23)$, and neuromuscular anomalies $(n=10)$. Seven categories with $<6$ cases were lumped into "others". The sporadic occurrence was customary compared to the familial presentation ( $\mathrm{n}=144$ vs 97). Across all families, the total number of affected individuals was 497 , with a preponderance of affected males compared to affected females (324 vs 173; Table 1).

Table 1. Major categories of CA, familial/sporadic nature, and total number of affected family members

\begin{tabular}{|c|c|c|c|c|c|c|c|c|c|c|}
\hline \multirow[t]{2}{*}{ Congenital anomalies } & \multicolumn{3}{|c|}{ Index subject } & \multirow[t]{2}{*}{ Proportion } & \multirow[t]{2}{*}{$95 \% \mathrm{Cl}$} & \multicolumn{2}{|c|}{$\begin{array}{c}\text { Familial/sporadic } \\
\text { nature* }^{*}\end{array}$} & \multicolumn{3}{|c|}{$\begin{array}{c}\text { Total no. of people affected } \\
\text { in all families* }\end{array}$} \\
\hline & Male & Female & Total & & & Familial & Sporadic & Male & Female & Total \\
\hline Limb defects & 89 & 24 & 113 & 0.469 & $0.406-0.532$ & 48 & 65 & 153 & 69 & 222 \\
\hline Neurological disorders & 57 & 19 & 76 & 0.315 & $0.257-0.374$ & 20 & 56 & 83 & 35 & 118 \\
\hline Musculoskeletal defects & 16 & 7 & 23 & 0.095 & $0.058-0.133$ & 8 & 15 & 23 & 18 & 41 \\
\hline Neuromuscular anomalies & 9 & 1 & 10 & 0.042 & $0.016-0.067$ & 5 & 5 & 21 & 2 & 23 \\
\hline Others $(n<6)$ & 10 & 9 & 19 & 0.079 & $0.045-0.113$ & 16 & 3 & 44 & 49 & 93 \\
\hline Total & 181 & 60 & 241 & 1.000 & - & 97 & 144 & 324 & 173 & 497 \\
\hline
\end{tabular}

${ }^{*}$ Chi-test statistics were statistically highly significant. 


\section{Limb defects}

Limb defects were further resolved into 16 distinct entities (Tables 2 and 3). Clubfoot had the highest representation $(n=26)$, followed by postaxial polydactyly type $A(n=23)$, preaxial polydactyly type $I(n=21)$, and arthrogryposis $(n=10)$. Certain defects, such as arthrogryposis, clubfoot, preaxial polydactyly type I, and reduction defects of upper limbs, mostly had sporadic presentation, whereas cases with postaxial polydactyly type A were more often familial. In all families with limb anomalies, a total of 222 subjects were observed to be affected.

Among the index cases with limb defects $(n=113)$, a total of 196 limbs were involved (Table 4). There was relatively higher involvement of the lower limbs compared to the upper limbs (109 vs 87) and of the right leg compared to the left leg (61 vs 48). Detailed analyses of the phenotypic variability and the combination of involved limbs are presented in Table 4.

\section{Neurological disorders}

Intellectual disability types (mild, severe, Down syndrome, and microcephaly) were in a sizable number and appeared in 35 subjects (Table 2). Cerebral palsy (CP) was the primary malformation in at least 32 subjects (and an associated malformation in further 18 individuals with intellectual disabilities; Table 5).

Table 2. Major and minor categories of congenital/hereditary malformations observed in the studied population

\begin{tabular}{|c|c|c|c|c|c|c|}
\hline Malformation (major/minor) & No. of cases & Proportion & $95 \% \mathrm{Cl}$ & ICD-10* & OMIM** & ORPHA\# \\
\hline Limb defects & 113 & 0.469 & $0.406-0.532$ & & & \\
\hline Clubfoot & 26 & 0.108 & $0.069-0.147$ & Q66 & 119800 & 293150,199315 \\
\hline Polydactyly, postaxial type A & 23 & 0.095 & $0.058-0.133$ & Q69.0;Q69.2 & 174200 & 93335 \\
\hline Polydactyly, preaxial type I & 20 & 0.083 & $0.048-0.118$ & Q69.1 & 174400 & 93339 \\
\hline Arthrogryposis & 10 & 0.042 & $0.016-0.067$ & Q74.3 & 108120 & 1146 \\
\hline Polydactyly, postaxial type B & 6 & 0.025 & $0.005-0.045$ & Q69.0;Q69.2 & 174200 & 93335 \\
\hline Reduction defects of upper limb & 6 & 0.025 & $0.005-0.045$ & Q71 & 217100 & 93457 \\
\hline Brachydactyly, 4th toe; brachymetatarsus IV & 4 & 0.017 & $0.001-0.033$ & Q72.8 & 113475 & 294998 \\
\hline Congenital shortening of lower limb & 4 & 0.017 & $0.001-0.033$ & Q72.8 & & 295057 \\
\hline Syndactyly, type 1a & 4 & 0.017 & $0.001-0.033$ & Q70.3 & 609815 & 93402,295187 \\
\hline Brachydactyly, all fingers short & 2 & 0.008 & -0.003 to 0.020 & Q71.8 & & 295130 \\
\hline Reduction defects of lower limb & 2 & 0.008 & -0.003 to 0.020 & Q72 & & 93457 \\
\hline Syndactyly, type 1c & 2 & 0.008 & -0.003 to 0.020 & Q70.1 & & 295191 \\
\hline Camptodactyly & 1 & 0.004 & -0.004 to 0.012 & & 114200 & 295016 \\
\hline Polydactyly, preaxial type II & 1 & 0.004 & -0.004 to 0.012 & Q74.0 & 174500 & 2950,93336 \\
\hline Split-hand/foot & 1 & 0.004 & -0.004 to 0.012 & Q72.7 & 183600 & 2440 \\
\hline Syndactyly, type II & 1 & 0.004 & -0.004 to 0.012 & Q70.4 & 186000 & 295195 \\
\hline Neurological disorders & 76 & 0.315 & $0.257-0.374$ & & & \\
\hline Intellectual disability: mild & 21 & 0.087 & $0.052-0.123$ & F70 & 249500 & 88616 \\
\hline Spastic diplegic cerebral palsy & 20 & 0.083 & $0.048-0.118$ & G80.1 & & \\
\hline Intellectual disability: severe & 11 & 0.046 & $0.019-0.072$ & F72 & 611091 & 88616 \\
\hline Hereditary neuropathy & 6 & 0.025 & $0.005-0.045$ & G60 & 201300 & 970 \\
\hline Cerebral palsy, unspecified & 4 & 0.017 & $0.001-0.033$ & G80.9 & 605388 & \\
\hline Spastic hemiplegic cerebral palsy & 3 & 0.012 & -0.002 to 0.026 & G80.2 & & \\
\hline Spastic quadriplegic cerebral palsy & 3 & 0.012 & -0.002 to 0.026 & G80.0 & 603513 & 210141 \\
\hline
\end{tabular}


Table 2. Major and minor categories of congenital/hereditary malformations observed in the studied population (Continued)

\begin{tabular}{|c|c|c|c|c|c|c|}
\hline Malformation (major/minor) & No. of cases & Proportion & $95 \% \mathrm{Cl}$ & ICD-10* & OMIM** & ORPHA\# \\
\hline Ataxic cerebral palsy & 2 & 0.008 & -0.003 to 0.020 & G80.4 & 605388 & \\
\hline Amyotrophic lateral sclerosis & 1 & 0.004 & -0.004 to 0.012 & G12.2 & 105400 & 803 \\
\hline Down syndrome & 1 & 0.004 & -0.004 to 0.012 & Q90.9 & 190685 & 870 \\
\hline Hereditary ataxia & 1 & 0.004 & -0.004 to 0.012 & $\mathrm{G} 11$ & 229300 & 95 \\
\hline Hereditary spastic paraplegia & 1 & 0.004 & -0.004 to 0.012 & G11.4 & 270800 & \\
\hline $\begin{array}{l}\text { Intellectual disability; succinic semialdehyde dehydro- } \\
\text { genase deficiency }\end{array}$ & 1 & 0.004 & -0.004 to 0.012 & & 271980 & 22 \\
\hline Microcephaly, low IQ^^ & 1 & 0.004 & -0.004 to 0.012 & Q02 & 251200 & 2512,52183 \\
\hline Musculoskeletal defects & 23 & 0.095 & $0.058-0.133$ & & & \\
\hline Achondroplasia & 12 & 0.050 & $0.022-0.077$ & Q77.4 & 100800 & 15 \\
\hline Kyphosis & 2 & 0.008 & -0.003 to 0.020 & Q76.4 & 192900 & \\
\hline Congenital deformity of forehead & 1 & 0.004 & -0.004 to 0.012 & Q75.8 & & \\
\hline Congenital dislocation of hip & 1 & 0.004 & -0.004 to 0.012 & Q65.2 & 142700 & \\
\hline Congenital malformation of bony thorax; genu valgum & 1 & 0.004 & -0.004 to 0.012 & Q76.9; Q74.1 & & \\
\hline Congenital scoliosis & 1 & 0.004 & -0.004 to 0.012 & Q76.3 & & \\
\hline Disorder of ligament (leg) & 1 & 0.004 & -0.004 to 0.012 & M24.2 & & \\
\hline Diastrophic dysplasia & 1 & 0.004 & -0.004 to 0.012 & Q77.5 & 222600 & 628 \\
\hline Genu valgum & 1 & 0.004 & -0.004 to 0.012 & Q44.1 & 137370 & \\
\hline Multiple congenital exostoses & 1 & 0.004 & -0.004 to 0.012 & Q78.6 & 133700 & 321 \\
\hline Osteochondrodysplasia, unspecified & 1 & 0.004 & -0.004 to 0.012 & Q78.9 & & \\
\hline Neuromuscular anomalies & 10 & 0.042 & $0.016-0.067$ & & & \\
\hline Muscular dystrophy & 8 & 0.033 & $0.011-0.056$ & G71.0 & 310200 & 98896 \\
\hline Spinal muscular atrophy & 2 & 0.008 & -0.003 to 0.020 & G12.1 & 253300 & 70,83330 \\
\hline Others & 19 & 0.079 & $0.045-0.113$ & & & \\
\hline Oculocutaneous albinism & 3 & 0.012 & -0.002 to 0.026 & E70.3 & 203100 & 79431,352731 \\
\hline Allergic asthma & 3 & 0.012 & -0.002 to 0.026 & J45.9 & & \\
\hline Deaf-mute & 3 & 0.012 & -0.002 to 0.026 & H91.3. & 220290 & 90636 \\
\hline Dysphasia & 1 & 0.004 & -0.004 to 0.012 & R47.0 & 600117 & \\
\hline Amblyopia & 1 & 0.004 & -0.004 to 0.012 & H53.0 & & \\
\hline Congenital malformation of cardiac septum & 1 & 0.004 & -0.004 to 0.012 & Q21.9 & 600001 & \\
\hline Deformed ear pinna & 1 & 0.004 & -0.004 to 0.012 & H61.9 & & 156243 \\
\hline Disorders of fluid, electrolyte, and acid-base balance & 1 & 0.004 & -0.004 to 0.012 & E87 & & \\
\hline High myopia & 1 & 0.004 & -0.004 to 0.012 & $\mathrm{H} 52.1$ & 160700 & \\
\hline Hypertensive heart disease & 1 & 0.004 & -0.004 to 0.012 & 111 & & \\
\hline Intestinal cancer & 1 & 0.004 & -0.004 to 0.012 & C17.9 & 26106 & \\
\hline Night blindness & 1 & 0.004 & -0.004 to 0.012 & H53.6 & 257270 & 215 \\
\hline Vitiligo & 1 & 0.004 & -0.004 to 0.012 & L80 & & \\
\hline
\end{tabular}

*ICD-10, International Classification of Diseases, Tenth Revision; $\wedge \mathrm{QQ}$, intelligence quotient; ${ }^{* *} \mathrm{OMIM}$, Online Mendelian Inheritance in Man; ORPHA, \#Orphanet database identifiers/Entrez. 
Table 3. Spectrum of limb defects and their distribution with respect to familial/sporadic nature and total number of affected family members

\begin{tabular}{|c|c|c|c|c|c|c|c|c|}
\hline \multirow{2}{*}{ Limb defects* } & \multicolumn{3}{|c|}{ Index cases } & \multicolumn{2}{|c|}{ Familial/sporadic } & \multicolumn{3}{|c|}{ Total no. of people affected in all families } \\
\hline & Male & Female & Total & Familial & Sporadic & Male & Female & Total \\
\hline Arthrogryposis & 8 & 2 & 10 & 2 & 8 & 10 & 8 & 18 \\
\hline Brachydactyly, brachymetatarsus IV & 4 & 0 & 4 & 3 & 1 & 8 & 4 & 12 \\
\hline Brachydactyly, all fingers & 0 & 2 & 2 & 1 & 1 & 1 & 2 & 3 \\
\hline Camptodactyly & 1 & 0 & 1 & 1 & 0 & 2 & 1 & 3 \\
\hline Clubfoot & 19 & 7 & 26 & 8 & 18 & 30 & 11 & 41 \\
\hline Congenital shortening of lower limb & 4 & 0 & 4 & 1 & 3 & 4 & 1 & 5 \\
\hline Polydactyly, postaxial type A & 19 & 4 & 23 & 13 & 10 & 35 & 16 & 51 \\
\hline Polydactyly, postaxial type B & 5 & 0 & 5 & 2 & 3 & 7 & 0 & 7 \\
\hline Polydactyly, preaxial type I & 17 & 4 & 21 & 8 & 13 & 25 & 13 & 38 \\
\hline Polydactyly, preaxial type II & 0 & 1 & 1 & 0 & 1 & 0 & 1 & 1 \\
\hline Reduction defects of lower limb & 2 & 0 & 2 & 1 & 1 & 2 & 1 & 3 \\
\hline Reduction defects of upper limb & 5 & 1 & 6 & 1 & 5 & 7 & 3 & 10 \\
\hline Split-hand/foot & 1 & 0 & 1 & 1 & 0 & 6 & 2 & 8 \\
\hline Syndactyly, type II & 0 & 1 & 1 & 1 & 0 & 1 & 2 & 3 \\
\hline Syndactyly, type 1a & 4 & 0 & 4 & 3 & 1 & 9 & 1 & 10 \\
\hline Syndactyly, type 1c & 0 & 2 & 2 & 2 & 0 & 6 & 3 & 9 \\
\hline Total & 89 & 24 & 113 & 48 & 65 & 153 & 69 & 222 \\
\hline
\end{tabular}

*Presented in alphabetical order.

Table 4. Phenotypic manifestation of limb defects $(n=113)$

\begin{tabular}{|c|c|c|c|c|c|c|c|c|c|c|c|c|c|}
\hline \multirow[t]{2}{*}{ Limb defects } & \multirow{2}{*}{$\begin{array}{l}\text { No. of } \\
\text { cases }\end{array}$} & \multirow{2}{*}{$\begin{array}{l}\text { Total no. of } \\
\text { affected limbs }\end{array}$} & \multicolumn{2}{|c|}{$\begin{array}{l}\text { Upper limb } \\
(n=87)\end{array}$} & \multicolumn{2}{|c|}{$\begin{array}{l}\text { Lower } \operatorname{limb} \\
(n=109)\end{array}$} & \multicolumn{3}{|c|}{$\begin{array}{l}\text { No. of cases with } \\
\text { involvement of .... }\end{array}$} & \multicolumn{4}{|c|}{$\begin{array}{c}\text { No. of limbs } \\
\text { involved }\end{array}$} \\
\hline & & & RA & LA & $\mathbf{R L}$ & LL & Arms only & Legs only & Both & Any 1 & Any 2 & Any 3 & All 4 \\
\hline Arthrogryposis & 10 & 22 & 6 & 7 & 5 & 4 & 5 & 1 & 4 & 3 & 4 & 1 & 2 \\
\hline $\begin{array}{l}\text { Brachydactyly, brachyme- } \\
\text { tatarsus IV }\end{array}$ & 4 & 5 & 0 & 0 & 3 & 2 & 0 & 4 & 0 & 3 & 1 & 0 & 0 \\
\hline Brachydactyly, all fingers & 2 & 4 & 2 & 2 & 0 & 0 & 2 & 0 & 0 & 0 & 2 & 0 & 0 \\
\hline Camptodactyly & 1 & 4 & 1 & 1 & 1 & 1 & 0 & 0 & 1 & 0 & 0 & 0 & 1 \\
\hline Clubfoot & 26 & 44 & 0 & 0 & 23 & 21 & 0 & 26 & 0 & 8 & 18 & 0 & 0 \\
\hline $\begin{array}{l}\text { Congenital shortening of } \\
\text { lower limb }\end{array}$ & 4 & 5 & 0 & 0 & 4 & 1 & 0 & 4 & 0 & 3 & 1 & 0 & 0 \\
\hline $\begin{array}{l}\text { Polydactyly, postaxial } \\
\text { type A }\end{array}$ & 23 & 50 & 12 & 12 & 16 & 10 & 3 & 9 & 11 & 9 & 6 & 3 & 5 \\
\hline $\begin{array}{l}\text { Polydactyly, postaxial } \\
\text { type B }\end{array}$ & 5 & 6 & 2 & 3 & 1 & 0 & 4 & 1 & 0 & 4 & 1 & 0 & 0 \\
\hline Polydactyly, preaxial type I & | 21 & 25 & 10 & 13 & 1 & 1 & 20 & 0 & 1 & 19 & 1 & 0 & 1 \\
\hline $\begin{array}{l}\text { Polydactyly, preaxial } \\
\text { type II }\end{array}$ & 1 & 1 & 1 & 0 & 0 & 0 & 1 & 0 & 0 & 1 & 0 & 0 & 0 \\
\hline $\begin{array}{l}\text { Reduction defects of } \\
\text { lower limb }\end{array}$ & 2 & 3 & 0 & 0 & 1 & 2 & 0 & 2 & 0 & 1 & 1 & 0 & 0 \\
\hline $\begin{array}{l}\text { Reduction defects of } \\
\text { upper limb }\end{array}$ & 6 & 7 & 4 & 3 & 0 & 0 & 6 & 0 & 0 & 5 & 1 & 0 & 0 \\
\hline Split-hand/foot & 1 & 4 & 1 & 1 & 1 & 1 & 0 & 0 & 1 & 0 & 0 & 0 & 1 \\
\hline Syndactyly, type II & 1 & 4 & 1 & 1 & 1 & 1 & 0 & 0 & 1 & 0 & 0 & 0 & 1 \\
\hline Syndactyly, type $1 a$ & 4 & 8 & 0 & 0 & 4 & 4 & 0 & 4 & 0 & 0 & 4 & 0 & 0 \\
\hline Syndactyly, type 1c & 2 & 4 & 2 & 2 & 0 & 0 & 2 & 0 & 0 & 0 & 2 & 0 & 0 \\
\hline Total & 113 & 196 & 42 & 45 & 61 & 48 & 43 & 51 & 19 & 56 & 42 & 4 & 11 \\
\hline
\end{tabular}

$L A$, left arm; $L L$, left leg; $R A$, right arm; $R L$, right leg 


\section{Associated malformations}

The secondary symptoms appearing with the primary presentation in the index subjects were scored as associated malformations. The combinations of associated malformations are depicted in Table 5. Limb anomalies were in the majority $(\mathrm{n}=23)$, followed by CP $(\mathrm{n}=18)$ and deaf-mute $(\mathrm{n}=8)$ cases.

\section{Familial cases}

The pedigrees with at least two affected subjects with a similar phenotypic presentation were considered as familial $(n=97)$. The analyses of pedigree structures revealed that the anomalies segregated in one or two generations in most of the cases ( $n=47$ and 34, respectively; data not shown). In the majority of pedigrees, the malformations appeared in two or one independent sibships ( $\mathrm{n}=45$ and 26, respectively).

\section{Parental consanguinity and parental ages}

Parental consanguinity was estimated to be $17 \%$ in the overall sample. The differences in the distribution of consanguineous and nonconsanguineous unions among the major categories of CA were statistically significant (Table 6). Consanguinity was relatively higher in subjects with neuromuscular anomalies and neurological disorders (30 and 21\%, respectively). Furthermore, consanguinity was significantly higher among familial cases compared to sporadic anomalies (29 vs $9 \%$, respectively; $P<0.0001$ ) (Table 6).

The differences in the mean paternal and maternal ages were statistically significant in all categories of sporadic and familial samples (Table 7). For the subjects with neuromuscular anomalies, the mean paternal ages were higher than for those with other anomalies.

The distribution of index subjects with respect to the demographic variables is presented in Table 8.

Table 5. Combinations of malformations associated with CA

\begin{tabular}{|c|c|c|c|c|c|c|c|c|}
\hline CA category & Brachydactyly & $\begin{array}{l}\text { Campto- } \\
\text { dactyly }\end{array}$ & $\begin{array}{c}\text { Syndactyly } \\
\text { type }\end{array}$ & Clubfoot & $\begin{array}{l}\text { Arthro- } \\
\text { gryposis }\end{array}$ & $\begin{array}{l}\text { Deaf- } \\
\text { mute }\end{array}$ & $\begin{array}{l}\text { Cerebral } \\
\text { palsy }\end{array}$ & Others \\
\hline Achondroplasia & & 1 & & & & 1 & & High myopia \\
\hline Arthrogryposis & & & III & & & & & \\
\hline Camptodactyly & First toe & & & & & & & \\
\hline Clubfoot & Fourth toe & 3 & $1 \mathrm{a}$ & & & & & \\
\hline Deaf-mute & & & & & & & & Polydactyly, postaxial type A \\
\hline Down syndrome & Fourth toe & & & & & & & Squint eye \\
\hline Dysphasia & & & & & 1 & & & \\
\hline Hereditary neuropathy & Third toe & & & & & & & Squint eye \\
\hline $\begin{array}{l}\text { Intellectual disability: } \\
\text { mild }\end{array}$ & & & & & 3 & 2 & 10 & Ataxia \\
\hline $\begin{array}{l}\text { Intellectual disability: } \\
\text { severe }\end{array}$ & Fourth toe & & & 1 & & 3 & 8 & $\begin{array}{c}\text { Cleft lip, overriding toe; } \\
\text { polydactyly postaxial type B }\end{array}$ \\
\hline Kyphosis & Fourth toe & & & 1 & & & & \\
\hline Muscular dystrophy & & & & & & & & Cleft lip \\
\hline $\begin{array}{l}\text { Polydactyly, postaxial } \\
\text { type A }\end{array}$ & & & $1 \mathrm{a}$ & & & & & Clinodactyly \\
\hline $\begin{array}{l}\text { Polydactyly, preaxial } \\
\text { type I }\end{array}$ & & 1 & & & & & & Hypoplastic thumb \\
\hline $\begin{array}{l}\text { Reduction defects of } \\
\text { lower limb }\end{array}$ & & & & 1 & 1 & & & \\
\hline $\begin{array}{l}\text { Spastic diplegic cerebral } \\
\text { palsy }\end{array}$ & Third toe & & & & & 1 & & Cleft palate \\
\hline Spinal muscular atrophy & & & & & & 1 & & \\
\hline Total & 7 & 5 & 3 & 3 & 5 & 8 & 18 & \\
\hline
\end{tabular}


Table 6. Relationship between consanguinity and different sample types

\begin{tabular}{|c|c|c|c|}
\hline \multirow{2}{*}{ Category } & \multicolumn{2}{|c|}{ Parental marriage type, $N(\%)$} & \multirow{2}{*}{ Total no. of marriages } \\
\hline & Consanguineous" & Nonconsanguineous & \\
\hline \multicolumn{4}{|l|}{ Malformation } \\
\hline Limb defects & $10(9)$ & $103(91)$ & 113 \\
\hline Neurological disorders & $16(21)$ & $60(79)$ & 76 \\
\hline Musculoskeletal defects & $4(17)$ & $19(83)$ & 23 \\
\hline Neuromuscular anomalies & $3(30)$ & $7(70)$ & 10 \\
\hline \multirow[t]{2}{*}{ Others } & $8(42)$ & $11(58)$ & 19 \\
\hline & & $P=0.003$ & \\
\hline \multicolumn{4}{|l|}{ Familial/sporadic nature } \\
\hline Familial & $28(29)$ & $69(71)$ & 97 \\
\hline Sporadic & $13(9)$ & $131(91)$ & 144 \\
\hline \multirow[t]{2}{*}{ Total } & $41(17)$ & $200(83)$ & 241 \\
\hline & & $P<0.0001$ & \\
\hline
\end{tabular}

"Inbreeding coefficient $F^{3} 0.0313$

Table 7. Mean parental ages at index subject's birth

\begin{tabular}{|c|c|c|c|c|}
\hline Category & $N$ & Paternal age (mean \pm SD) & Maternal age (mean \pm SD) & Significance level $(P)^{\#}$ \\
\hline \multicolumn{5}{|l|}{ Sporadic cases $(n=137)$} \\
\hline Limb defects & 62 & $33.4 \pm 5.0$ & $27.0 \pm 5.2$ & $<0.0001$ \\
\hline Neurological disorders & 56 & $32.8 \pm 4.8$ & $26.3 \pm 4.6$ & $<0.0001$ \\
\hline Musculoskeletal defects & 14 & $35.8 \pm 4.9$ & $29.3 \pm 4.7$ & 0.0014 \\
\hline Neuromuscular anomalies & 5 & $40.0 \pm 3.7$ & $32.8 \pm 3.6$ & 0.0143 \\
\hline \multirow[t]{2}{*}{ Total } & 137 & $33.7 \pm 5.1$ & $27.1 \pm 5.0$ & $<0.0001$ \\
\hline & & ANOVA, $P=0.910$ & ANOVA, $P=0.726$ & \\
\hline \multicolumn{5}{|l|}{ Familial cases $(n=70)$} \\
\hline Limb defects & 43 & $34.7 \pm 7.4$ & $27.7 \pm 6.2$ & $<0.0001$ \\
\hline Neurological disorders & 16 & $31.8 \pm 5.6$ & $25.4 \pm 4.8$ & 0.0018 \\
\hline Musculoskeletal defects & 7 & $31.4 \pm 5.6$ & $25.3 \pm 5.9$ & 0.0702 \\
\hline Neuromuscular anomalies & 4 & $36.0 \pm 6.8$ & $29.0 \pm 5.0$ & 0.1469 \\
\hline \multirow[t]{2}{*}{ Total } & 70 & $33.8 \pm 6.9$ & $27.0 \pm 5.9$ & $<0.0001$ \\
\hline & & ANOVA, $P=0.561$ & ANOVA, $P=0.689$ & \\
\hline
\end{tabular}

"T-test statistics were used for comparison between paternal and maternal ages.

One-way analysis of variance (ANOVA) was used for analysis among the paternal ages or the maternal ages.

Table 8. Demographic characteristics of index subjects

\begin{tabular}{|c|c|c|c|c|c|}
\hline \multirow{2}{*}{ Demographic variables } & \multicolumn{2}{|c|}{ Gender } & \multicolumn{2}{|c|}{ Familial/sporadic } & \multirow[t]{2}{*}{ Total } \\
\hline & Male & Female & Familial & Sporadic & \\
\hline \multicolumn{6}{|l|}{ Age category, years } \\
\hline$\leq 9$ & 26 & 19 & 16 & 29 & 45 \\
\hline$>9-19$ & 67 & 28 & 28 & 67 & 95 \\
\hline$>19-29$ & 40 & 5 & 22 & 23 & 45 \\
\hline$>29-39$ & 22 & 4 & 15 & 11 & 26 \\
\hline$\geq 39$ & 26 & 4 & 16 & 14 & 30 \\
\hline \multirow[t]{2}{*}{ Total } & 181 & 60 & 97 & 144 & 241 \\
\hline & \multicolumn{2}{|c|}{$P=0.0027$} & \multicolumn{2}{|c|}{$P=0.0187$} & \\
\hline
\end{tabular}


Table 8. Demographic characteristics of index subjects (Continued)

\begin{tabular}{|c|c|c|c|c|c|}
\hline \multirow{2}{*}{ Demographic variables } & \multicolumn{2}{|c|}{ Gender } & \multicolumn{2}{|c|}{ Familial/sporadic } & \multirow[t]{2}{*}{ Total } \\
\hline & Male & Female & Familial & Sporadic & \\
\hline \multicolumn{6}{|l|}{ Rural/urban origin } \\
\hline Rural & 148 & 45 & 72 & 121 & 193 \\
\hline \multirow[t]{2}{*}{ Urban } & 33 & 15 & 25 & 23 & 48 \\
\hline & & $P=0.2553$ & & $P=0.0617$ & \\
\hline \multicolumn{6}{|l|}{ Caste system } \\
\hline Jatt & 51 & 12 & 32 & 31 & 63 \\
\hline Rajput & 32 & 7 & 13 & 26 & 39 \\
\hline Arain & 18 & 7 & 9 & 16 & 25 \\
\hline Malik & 13 & 9 & 10 & 12 & 22 \\
\hline Meher & 14 & 4 & 10 & 8 & 18 \\
\hline \multirow[t]{2}{*}{ Others } & 53 & 21 & 23 & 51 & 74 \\
\hline & & $P=0.3207$ & & $P=0.1325$ & \\
\hline \multicolumn{6}{|l|}{ Migratory origin" } \\
\hline Native & 150 & 42 & 76 & 116 & 192 \\
\hline \multirow[t]{2}{*}{ Migrated } & 31 & 18 & 21 & 28 & 49 \\
\hline & & $P=0.0318$ & & $P=0.6766$ & \\
\hline \multicolumn{6}{|l|}{ Literacy (age > 5 years) } \\
\hline Illiterate & 98 & 31 & 49 & 80 & 129 \\
\hline \multirow[t]{2}{*}{ Literate } & 71 & 21 & 41 & 51 & 92 \\
\hline & & $P=0.8351$ & & $P=0.3263$ & \\
\hline \multicolumn{6}{|l|}{ Family type } \\
\hline Nuclear & 107 & 30 & 67 & 70 & 137 \\
\hline \multirow[t]{2}{*}{ Extended } & 74 & 30 & 30 & 74 & 104 \\
\hline & & $P=0.2166$ & & $P=0.0017$ & \\
\hline
\end{tabular}

"During the 1947 partition.

\section{Discussion}

Pakistan bears a high burden of congenital and hereditary anomalies. The health-care system is not able to provide management and support to the subjects/families afflicted with such anomalies, resulting in a great social, economic, and psychological impact on the involved families and the society at large. In Pakistan, 6\%-9\% perinatal deaths are due to CA [24]. Many of the known risk factors, such as advanced maternal age, exposure to teratogens and radiation, maternal illnesses, and smoking, could be substantially minimized by educating pregnant women and providing them timely antenatal care. Furthermore, various screening methods, such as determination of maternal serum markers, ultrasonography, amniocentesis, and chorionic villus sampling, can be utilized to detect the at-risk pregnancies and their subsequent management. In this context, basic epidemiological data provide useful grounds for assigning priorities, allocating resources, and establishing monitoring and management systems for these disorders.

Limb defects had the highest representation in the present study, 113 cases with primary presentation and at least 28 cases with associated defects. Polydactyly types were most prevalent, followed by clubfoot, arthrogryposis, reduction defects, and syndactyly types. Most of the limb/digit defects do not cause severe disability and, owing to their minor nature, such anomalies remain less reported in epidemiological studies [12, $25,26]$. As witnessed in the present cohort, the majority of limb defects were of milder nature and did not result in any disability. However, there were at least 45 cases that were the potential sources of disability (including clubfoot $=26$; arthrogryposis $=10$; reduction defects of upper $\operatorname{limb}=6$; reduction defects of lower limb $=2$; split-hand/foot $=1$ ). In a study carried out in a tribal area of Pakistan, Zahra et al. [27] demonstrated that limb defects were the third most common anomalies (21\%), after neurological disorders (34\%) and musculoskeletal defects $(23 \%)$. Curiously, amputations/reduction defects were the most common types among limb anomalies. Polydactyly was also witnessed to be the most common type of limb anomaly in other studies [25, 28].

Among the neurological disorders, $\mathrm{CP}$ was a major source of severe disability in our cohort. $\mathrm{CP}$ is a chronic motor disorder that is nonprogressive in nature. Subjects with CP may 
have several problems, including walking disability, hearing and eye problems, seizures, feeding problems, and intellectual disability $[29,30]$. In our data, different clinical types of CP were recognized, including hemiplegia, diplegia, quadriplegia, and ataxia. There are several other conditions that should be considered in the differential diagnosis of CP. For instance, diplegic CP (OMIM-605388) resembles hereditary spastic paraplegia (HSP). However, $\mathrm{CP}$ is nonprogressive, whereas HSP is characterized by a steady weakness of the lower limbs. ${ }^{31}$ On the other hand, in muscular dystrophies, there is no spasticity, yet the patients can develop contractures. In primary dystonia (OMIM-128100), which is another movement disorder, the onset of muscular deformity occurs after several years of normal development. The patients have sustained episodes of muscle contraction and dystonia but without the development of contractures. Hereditary spastic paraparesis type 4 (OMIM-182601) is the single most common dominantly inherited paraparesis, representing approximately $40 \%$ of all cases [32].

Among the musculoskeletal anomalies, achondroplasia was predominant $(n=12)$ in the present cohort, followed by other disorders, including kyphosis, deformities of the hip, and disorders of the ligament, which were also the causes of physical disability. Another source of disability among the subjects was the occurrence of neuromuscular disorders, represented by muscular dystrophy $(\mathrm{n}=8)$ and spinal muscular atrophy $(\mathrm{n}=2)$. Azhar et al [33]. carried out an interventional study on disabled subjects in Sialkot District. Among the 644 individuals with disabilities, poliomyelitis was a predominant disorder, followed by CP, skeletal dysplasias, muscular dystrophy, congenital dislocated hip, and talipes equinovarus. The cases of poliomyelitis were excluded from our sample $(n=7)$. Nonetheless, the primary focus of Azhar et al. [33] was on the recovery of disabled individuals by surgical interventions, physiotherapy, and bracing, and no attempt was made to describe the nature and clinical spectrum of anomalies. The current study, however, presents a range of phenotypes for these anomalies.

Parental consanguinity was calculated to be $17 \%$ in the present cohort. Among the subjects with neuromuscular anomalies, the rate of consanguinity was $30 \%$. This is rather surprising as several studies have advocated a high incidence of consanguineous marriages, that is, $57-62 \%$, in various Pakistani populations [34-36]. Nonetheless, the increased incidence of CA is generally attributed to a high rate of parental consanguinity in Pakistani society [11]. The low rate of consanguinity may indicate the involvement of nongenetic factors in the etiology of these anomalies.

In this study, the high incidence of limb and neurological disorders, the preponderance of cases with sporadic nature, and the relatively low level of parental consanguinity may indicate a substantial contribution of environmental factors in the etiology of CA. Gao et al. [37] in a case-control study demonstrated that several factors, including advanced maternal age, alcohol consumption during pregnancy, rural residence, father's occupational exposure to harmful substances, and multiple births, were important risk factors for $\mathrm{CP}$ in Chinese children. Prenatal exposure to environmental factors was reported to cause congenital limb defects [38]. Furthermore, agricultural compounds in water were observed to be the source of birth defects, which include limb anomalies [39].

There could be several potential nongenetic causes of the high prevalence of limb and neurological anomalies in the study region, for instance, poor prenatal care, lack of basic health facilities, and maternal exposures. In the rural areas of Pakistan, primary maternity care is provided by traditional midwives/birth attendants, who are not professionally trained and cannot handle birth complications [15]. Birth defects such as CP may appear in newborns due to birth asphyxia. Second, Sialkot is an industrial city that is famous for its leather, sports, and surgical products. With 117 operational tanning units, Sialkot is the second largest tanning cluster of Punjab Province. Only 3\% of industrial plants meet international waste treatment standards [17]. A large number of industries discharge their toxic waste into natural watercourses and open streams [40, 41]. The industrial waste is used to irrigate vegetable and fruit farms [42]. Third, due to the adjoining boundary with India, the rural population is continuously threatened by cross-border shelling, which may add heavy metals in the human food chain. Thus, Sialkot population is constantly exposed to teratogens, such as heavy metals, which could be a potential cause for the high incidence of CA in the region. However, a direct association between environmental exposures and CA incidence remains to be established in this population.

Similar to other epidemiological studies, the current study has several limitations [25, 27, 28]. For instance, the exact prevalence rate of CA was not established in this study. This study presents CA cases that were rather explicit in nature and could be easily phenotyped with the physical examination, notwithstanding the fact that detailed clinical data, including X-rays and other laboratory investigations, were available for many of the cases/families. Maladies of biochemical or metabolic nature or those that require invasive diagnostic methods (such as structural brain defects) could be underrepresented in our cohort. Furthermore, there was no exhaustive coverage of areas of the whole district due to sociocultural limitations and unavailability of resource persons. 


\section{Conclusion}

This preliminary study in Sialkot District presents a detailed clinical and descriptive account of CA prevalent in this population. High incidence of limb and neurological anomalies, low level of parental consanguinity, and the preponderance of sporadic cases in this cohort suggest a significant role of nongenetic etiological factors, which could be minimized by strengthening the health-care system.

Author contributions. Sajid M contributed to the conception and design of this study. NAB collected the data and drafted the initial version of the manuscript. Sara M and Sajid M critically revised it and completed the write-up. All the authors approved the final version submitted for publication and take responsibility for statements made in the published article.

Acknowledgments. We sincerely acknowledge the participation of subjects/families in this study. The help of resident officers and specialized doctors at district hospitals of Sialkot is highly appreciated. This work was supported by the URF, Islamabad.

Conflict of interest statement. The authors have completed and submitted the International Committee of Medical Journal Editors Uniform Disclosure Form for Potential Conflicts of Interest. None of the authors disclose any conflict of interest.

\section{References}

[1] Center for Disease Control and Prevention [online]. 2016 [cited 2017 Jan 27]. Available from: https://www.cdc.gov/

[2] World Health Organization. Congenital anomalies, Factsheet [online]. 2016. [cited 2017 Jan 27]. Available from: http://www.who. int/mediacentre/factsheets/fs370/en/

[3] World Health Organization. Neonatal-perinatal database and birth defects surveillance. Report of the regional review meeting, New Delhi, India, 19-21 August 2014.

[4] EUROCAT. European Surveillance of Congenital Anomalies [online]. 2015. [cited 2017 Jan 17]. Available from: http://www. eurocat-network.eu/

[5] Abdolahi HM, Maher MHK, Afsharnia F, Dastgiri S. Prevalence of congenital anomalies: a community-based study in the northwest of Iran. ISRN Pediatr. 2014; 920-40.

[6] Ndibazza J, Lule S, Nampijja M, Mpairwe H, Oduru G, Kiggundu $\mathrm{M}$, et al. A description of congenital anomalies among infants in Entebbe, Uganda. Birth Defects Res A Clin Mol Teratol. 2011; 91:857-61.

[7] Kumar S, Bano S. Comparison and analysis of health care delivery systems: Pakistan versus Bangladesh. J Hosp Med Manage. 2017; 3:1.

[8] Hall JJ, Taylor R. Health for all beyond 2000: the demise of the Alma-Ata Declaration and primary health care in developing countries. Med J Aust. 2003; 178:17-20.
[9] United Nations, Department of Economic and Social Affairs, Population Division. 2015; World Population Prospects: The 2015 Revision.

[10] Arfaksad A, Wajahat Y. 2015. Frequency of fetal congenital anomalies and associated risk factors observed in pregnant women in a public sector hospital of Karachi. Ann Abbasi Shaheed Hosp Karachi Med Dent Coll. 2016; 21:139-46.

[11] Masood SN, Jamil N, Mumtaz SN, Masood MF, Muneer S. Congenital malformations in newborns of consanguineous and non-consanguineous parents. Pak J Med Sci. 2011; 27:1-5.

[12] Gillani S, Kazmi NHS, Najeeb S, Hussain S, Raza A. Frequencies of congenital anomalies among newborns admitted in nursery of Ayub Teaching Hospital Abbottabad, Pakistan. J Ayub Med Coll Abbottabad. 2011; 23:117-21.

[13] Hussain S, Sabir MU, Tarar SH, Mushtaq R. Prevalence and pattern of congenital malformations among neonates in the neonatal unit of a teaching hospital. J Pak Med Assoc. 2014; 64:629-34.

[14] Perveen F, Tyyab S. Frequency and pattern of distribution of congenital anomalies in the newborn and associated maternal risk factors. J Coll Physicians Surg Pak. 2007; 17:340-3.

[15] Shah N, Rohra DK, Shams H, Khan NH. Home deliveries: reasons and adverse outcomes in women presenting to a tertiary care hospital. J Pak Med Assoc. 2010; 60:555-8.

[16] National Institute of Population Studies (NIPS) [Pakistan] and ICF International. 2013. Pakistan Demographic and Health Survey 2012-13. Islamabad, Pakistan, and Calverton, Maryland, USA: NIPS and ICF International. [online] 2014. [cited 2017 Feb 05]. Available from: https://www.nips.org.pk/abstract_files/PDHS\%20Final\%20 Report\%20as\%20of\%20Jan\%2022-2014.pdf.

[17] District Disaster Management Authority. Disaster Risk Management Plan. District Sialkot, Government of Punjab. DCO Office Sialkot [online]. 2008. [cited 2017 Jan 27]. Available from: http//www.ndma. gov.pk

[18] International Classification of Disease. [online] 2016. [cited 2017 Jan 15]. Available from: http://apps.who.int/classifications/icd10/ browse/2016/en

[19] Online Mendelian Inheritance in Man. [online] 2016. [cited 2017 Feb 05]. Available from: http://www.ncbi.nlm.nih.gov/pubmed

[20] ORPHANET. The portal for rare diseases and orphan drugs. [online] 2017. [cited 2017 Feb 13]. Available from: http://www.orpha.net/

[21] Temtamy SA, McKusick VA. The genetics of hand malformations. New York: Alan R. Liss; 1978.

[22] Malik S. Syndactyly: phenotypes, genetics and current classification. Eur J Hum Genet. 2012; 20:817-24.

[23] Malik S. Polydactyly: phenotypes, genetics and classification. Clin Genet. 2014; 85:203-12.

[24] Korejo R, Bhutta S, Noorani KH, Bhutta ZA. An audit and trends of perinatal mortality at the Jinnah Postgraduate Medical Centre Karachi. J Pak Med Assoc. 2007; 57:168-71.

[25] Lal K, Malik S. Epidemiological study of congenital limb defects in individuals or families from the interior Sindh region of Pakistan. Asian Biomed (Res Rev News). 2015; 9:325-34.

[26] Khan A, Zuhaid M, Fayaz M, Ali F, Khan A, Ullah R, et al. Frequency of congenital anomalies in newborns and its relation to maternal health in a tertiary care hospital in Peshawar. Pakistan. Int J Med Students. 2015; 3:19-23.

[27] Zahra Q, Shuaib M, Malik S. Epidemiology of congenital anomalies in the Kurram Tribal Agency, northwest Pakistan. Asian Biomed (Res Rev News). 2016; 10:591-601. 
[28] Ullah S, Dasti JI, Malik S. Descriptive epidemiology of hereditary musculoskeletal and limb defects in the isolated population of Chitral, North-West Pakistan. Pak J Med Sci. 2015; 31:1047-52.

[29] Jan MM. Cerebral palsy: comprehensive review and update. Ann Saudi Med. 2006; 26:123-32.

[30] O'Shea TM. Diagnosis, treatment, and prevention of cerebral palsy. Clin Obstet Gynecol. 2008; 51:816-28.

[31] McDermott C, White K, Bushby K, Shaw P. Hereditary spastic paraparesis: a review of new developments. J Neurol Neurosurg Psychiatry. 2000; 69:150-60.

[32] Fink JK. Hereditary spastic paraplegia overview. In: Pagon RA, Adam MP, Ardinger HH, editors. GeneReviews ${ }^{\oplus}$ [Internet]. Seattle: University of Washington; 1993-2017.

[33] Azhar MM. Helping the disabled to become respectable members of the society. Pak J Med Health Sci. 2011; 5:720-3.

[34] Jabeen N, Malik S. Consanguinity and its sociodemographic differentials in Bhimber District, Azad Jammu and Kashmir, Pakistan. J Health Popul Nutr. 2014; 32:301-13.

[35] Hina S, Malik S. Pattern of consanguinity and inbreeding coefficient in Sargodha district, Punjab, Pakistan. J Biosoc Sci. 2015; 47:803-11
[36] Riaz HF, Mannan S, Malik S. Consanguinity and its socio-biological parameters in Rahim Yar Khan District, Southern Punjab, Pakistan. J Health Pop Nutrition. 2016; 35:14.

[37] Gao J, Zhao B, He L, Sun M, Yu X, Wang L. Risk of cerebral palsy in Chinese children: AN:M matched case control study. J Paediatr Child Health. 2017; 53:464-9.

[38] Alexander PG, Clark KL, Tuan RS. Prenatal exposure to environmental factors and congenital limb defects. Birth Defects Res C Embryo Today. 2016; 108:243-73.

[39] Brender JD, Weyer PJ. Agricultural compounds in water and birth defects. Curr Environ Health Rep. 2016; 3:144-52.

[40] Mahmood-ul-Hassan M, Suthor V, Rafique E, Ahmad R, Yasin M. Metal contamination of vegetables grown on soils irrigated with untreated municipal effluent. Bull Environ Contam Toxicol. 2012; 88:204-9.

[41] Khan MU, Malik RN, Muhammad S, Ullah F, Qadir A. Health risk assessment of consumption of heavy metals in market food crops from Sialkot and Gujranwala districts, Pakistan. Hum Ecological Risk Assessment. 2015; 21:327-37.

[42] Junaid M, Hashmi MZ, Malik RN. Evaluating levels and health risk of heavy metals in exposed workers from surgical instrument manufacturing industries of Sialkot, Pakistan. Environ Sci Pollut Res Int. 2016; 23:18010-26. 